\title{
Considérations
}

\section{sur le genre Nycteria (Haemoproteidae) parasite de Microchiroptères africains avec description de quatre espèces nouvelles ${ }^{(1)}$}

\author{
par G. ROSIN *, I. LANDAU ** et J.-P. HUGOT *** \\ * Laboratoire de Parasitologie, C.H.U. Henri-Mondor, 94010 Créteil. \\ *中. Laboratoire de Zoologie (Vers) associé au C.N.R.S., \\ Muséum national d'Histoire naturelle, 43, rue Cuvier, F 75231 Paris Cedex 05. \\ thow Centre de Primatologie, C.N.R.S., B.P. 181, Makokou, Gabon.
}

\section{Résumẹé.}

Cinq espèces de Nycteria ont été étudiées en Afrique centrale (Gabon et Congo-Brazzaville). Elles se répartissent en 2 groupes distincts: les parasites de Nycteris ayant des gaméiocytes « en cocarde »: $N$. medusiformis, $N$. houini n. sp. et $N$. erardi n. sp, ; les parasites de Rhinolophus qui ont des gamétocytes «diffus $\diamond: N$. gabonensis $\mathrm{n}$. sp. et $N$. krampitzi $\mathrm{n}$. sp.

Les caractères du genre Nycteria et sa place parmi les autres Hémosporidies sont analysés.

\section{Summary.}

Considerations on the genus Nycteria (Haemoproteidae) parasite of African Microchiroptera with the description of 4 new species.

5 species of Nycteria from Central Airica (Gabon and Congo-Brazzaville) were studied. They can be divided into two distinct groups : the parasites of Nycteris with gametocytes « en cocarde »: $N$. medusiformis, $N$. houini n. sp. and $N$. erardi n. sp. ; the parasites of Rhinolophus with gametocytes of the "diffus » type : N. gabonensis n. sp. and N. krampitzi n. sp.

The characteristics of the genus Nycteria and its place amongst the other Haemosporidia are analysed.

Accepté le 30 janvier 1978.

(1) Travail effectué grâce à une subvention de l'O.M.S. 
Trois genres d'Haemoproteidae sont connus chez les Microchiroptères :

1) Hepatocystis Levaditi et Schoen, 1932, qui n'a jusqu'à présent été signalé qu'en Asie (Manwell, 1966 et Landau, Miltgen, Le Bail et Yap, 1976). Il a des gamétocytes de type vivax, tantôt «diffus», tantôt «en cocarde », (Landau, Miltgen et Chabaud, 1976) et des schizontes de grande taille contenant des amas plus ou moins importants de colloïde. Les Culicoïdes en sont probablement les vecteurs.

2) Polychromophilus Dionisi, 1899, parasite cosmopolite atteignant de nombreuses espèces de Chauve-souris dans le monde entier; il est transmis par des Nyctéribies et se caractérise par des gamétocytes de forme ovalaire dans les frottis séchés lentement et qui semblent plus proches des gamétocytes d'Haemoproteidae d'Oiseaux ou de Reptiles que de ceux des Mammifères.

Ils ont deux types de schizontes tissulaires (Landau et coll., 1978) : des microschizontes disséminés dans les viscères et des macroschizontes pulmonaires.

3) Nycteria Garnham et Heisch, 1953, qui regroupe des parasites de Nycteris et Rhinolophus en Afrique tropicale et sub-tropicale. Les gamétocytes sont de type malariae. La schizogonie tissulaire évolue dans les cellules du parenchyme hépatique; les schizontes, de taille relativement petite, se rapprochent plus des formes pré-érythrocytaires des Plasmodium de Mammifères que de celles d'Hepatocystis.

Dans cet article, nous décrivons (2) trois espèces nouvelles de Nycteria du Gabon : Nycteria houini n. sp., parasite de Nycteris nana (Anderson, 1912), dédiée à R. Houin ; Nycteria erardi n. sp., parasite de Nycteris arge (Thomas, 1903), dédiée à C. Erard ; Nycteria gabonensis n. sp., parasite de Rhinolophus sylvestris (Aellen, 1959).

Une quatrième espèce parasite d'un Rhinolophus sp. du Congo a déjà été décrite par Landau en 1973 sous le nom d'Hepatocystis sp. Il s'agit, en fait, d'une Nycteria (3) ; nous rappelons ici ses principaux caractères et la nommons Nycteria krampitzi n. sp. (= Hepatocystis sp. Landau, 1973 pro parte), en hommage à H. E. Krampitz.

Nycteria medusiformis a été retrouvé chez plusieurs Nycteris grandis (Peters, 1865) du Gabon; nous le redécrivons brièvement ici à partir de ce nouveau matériel.

Les principaux caractères taxonomiques du genre Nycteria seront développés à la fin de cet article et les affinités du genre avec les Plasmodium seront discutées.

Le détail des lieux et dates de capture, ainsi que le nombre d'animaux parasités, sont rassemblés dans le tableau I.

(2) Le matériel type est déposé au M.N.H.N. sous les nos: N. houini: P. VI 132, 138 à 142 ; $N$. erardi: P. VI 131,143 à $147 ; N$. congolensis: P. VI 133,$148 ; N$. krampitzi : P. III 120 à 124 , 139 à 142.

(3) Sous le nom d'Hepatocystis sp., Landau, 1973 a englobé des formes parasitaires trouvées chez deux genres de Chiroptères: Miniopterus minor et Rhinolophus sp.; Nycteria krampitzi comprend tous les stades (gamétocytes et schizontes) trouvés chez Rhinolophus sp. 
Tableau I. Conditions de capture et nombre d'animaux parasités

\begin{tabular}{|c|c|c|c|c|c|c|}
\hline Hôtes & Lieu de capture & Biotope & $\begin{array}{l}\text { Date } \\
\text { de capture }\end{array}$ & $\begin{array}{l}\text { Animaux } \\
\text { e examinés }\end{array}$ & $\begin{array}{l}\text { Animaux } \\
\text { parasités }\end{array}$ & Saison \\
\hline Nycteris grandis & $\begin{array}{l}\text { Makokou } \\
\text { (Gabon) }\end{array}$ & $\begin{array}{l}\text { Buses } \\
\text { sous une } \\
\text { route }\end{array}$ & $\begin{array}{l}14 \text { au } \\
30-7-77\end{array}$ & 7 & 4 & grande saison sèche \\
\hline Nycteris arge .. & $\begin{array}{l}\text { Makokou } \\
\text { (Gabon) }\end{array}$ & Forêt & $10-2-77$ & 1 & 1 & petite saison sèche \\
\hline Nycteris nana .. & $\begin{array}{l}\text { Bengoue } \\
\text { (Gabon) }\end{array}$ & $\begin{array}{l}\text { Galerie } \\
\text { de mine }\end{array}$ & $25-7-76$ & 1 & 1 & grande saison sèche \\
\hline $\begin{array}{l}\text { Rhinolophus } \\
\text { sylvestris } \ldots \ldots\end{array}$ & $\begin{array}{l}\text { Bellinga } \\
\text { (Gabon) }\end{array}$ & $\begin{array}{l}\text { Galerie } \\
\text { de mine }\end{array}$ & $\begin{array}{l}23-7-77 \\
4-12-76\end{array}$ & $\begin{array}{l}21 \\
11\end{array}$ & $\begin{array}{l}4 \\
6\end{array}$ & $\begin{array}{l}\text { grande saison sèche } \\
\text { saison des pluies }\end{array}$ \\
\hline Rhinolophus sp. & $\begin{array}{c}\text { Meya } \\
\text { (Congo } \\
\text { Brazzaville) }\end{array}$ & Grotte & $23-2-67$ & 2 & 2 & grande saison sèche \\
\hline
\end{tabular}

Nous remercions A. Brosset pour le matériel qu'il nous a fourni et pour les déterminations de Chauve-souris, ainsi que M.-C. Durette-Desset pour les captures de Rhinolophus sylvestris en décembre 1976.

Les frottis sanguins ont été fixés par le méthanol et colorés par le Giemsa; les pièces histologiques ont été fixées par le Carnoy et colorées par le Giemsa colophane.

\section{A. - DESCRIPTIONS.}

\section{I. - Nycteria bouini n. sp.}

- Gamétocytes (Pl. I, 1, 2):

Les gamétocytes gardent tout au long de leur évolution une forme circulaire et compacte.

Les formes très jeunes ont un cytoplasme peu colorable, bleu pâle. A ce stade, une petite vacuole allongée sépare le noyau du cytoplasme, qui ne contient pas encore de pigment. Le noyau unique est bien délimité ; en forme de croissant, il possède une chromatine homogène. Le cytoplasme s'épaissit progressivement et se teinte en bleu plus soutenu. D'apparition précoce, rassemblé en un amas périphérique, le pigment est fin, jaune et abondant. Il prendra ensuite une teinte noirâtre, devenant grossier et dispersé.

Les micro et les macrogamétocytes sont ronds et de même taille; ils mesurent 7,5 $\mu \mathrm{m}$ en moyenne dans les parties minces et bien étalées des frottis ; ils n'envahissent 
pas toute la cellule-hôte, qui reste apparente, sous forme d'une auréole ou d'un fragment polaire fortement teinté en rose. Cette coloration foncée de l'hématie est frappante. Le macrogamétocyte a un cytoplasme granulaire, bleu soutenu, qui est le seul caractère permettant de le différencier du microgamétocyte, qui est bleu pâle ou gris clair homogène. Les noyaux dans les deux sexes sont identiques, avec une partie centrale arrondie ou ovalaire bien limitée, à chromatine dense et homogène. Le pigment est, dans les deux cas, constitué par 10 à 16 grains noirâtres, évoquant des grains de riz irrégulièrement distribués dans le cytoplasme.

Enfin, le microgamétocyte exflagelle en moins de deux minutes.

- Schizontes (Pl. II, 2, 3, 4):

Sept schizontes à différents stades de maturation ont été observés dans le foie. Aucune forme n'a été trouvée dans le poumon, la rate et le rein.

Le plus jeune schizonte observé mesure $28 \mu \mathrm{m}$ sur $16 \mu \mathrm{m}$; il est entouré par une membrane rose relativement épaisse $(1,5 \mu \mathrm{m})$; son cytoplasme est granuleux, très basophile et chromophile, et contient en périphérie quelques globules de collö̈de. Les noyaux sont de grande taille et ont des contours irréguliers. Ils sont uniformément distribués dans le schizonte, sauf à la périphérie, où une bande circulaire en est dépourvue. En mûrissant, leur forme reste ovoïde et régulière. Ils présentent une membrane fine et parfois, dans la masse nucléocytoplasmique, une incisure séparant deux lobes. Le cytoplasme devient plus clair, et les noyaux plus petits. Nous n'avons pas observé de forme complètement mûre, mais 2 schizontes paraissaient proches de la maturité. Ils mesurent $46 \mu \mathrm{m} \times 27,5 \mu \mathrm{m}$ et $39 \mu \mathrm{m} \times 21 \mu \mathrm{m}$. Les noyaux, nombreux et petits, forment des travées délimitant des vacuoles qui confèrent au parasite un aspect lacunaire très caractéristique. Ils déterminent une faible réaction tissulaire, constituée par une assise de cellules macrophagiques. Il semble que la formation de cytomères et la réaction macrophagique soient liées, aucun autre schizonte sans cytomère n'étant attaqué par les macrophages.

\section{II. - Nycteria erardi n. sp.}

\section{- Gamétocytes (Pl. I, 9) :}

Les plus jeunes formes observées sont circulaires et compactes sans vacuole. Le cytoplasme bleu pâle est homogène. Le pigment est déjà présent, constitué par un ou deux grains jaune verdâtre ; nous n'avons trouvé aucun stade qui en soit dépourvu. Le noyau est constitué par plusieurs granules ronds (2 à 4) reliés entre eux par des ponts de chromatine. A mesure que le gamétocyte évolue, sa forme générale reste arrondie. Le noyau devient central, perd son aspect granuleux, et la chromatine forme une seule masse aux contours irréguliers. Le pigment, bien différencié chez ces formes encore jeunes, comporte un nombre limité de gros grains noirâtres en bâtonnets (9 à 13 grains). 
A maturité, les deux sexes sont de taille égale (7,2 $\mu \mathrm{m}$ en moyenne). Les microgamétocytes sont de forme circulaire; ils n'envahissent pas la totalité de l'hématie hôte. Le cytoplasme des gamétocytes mâles est homogène bleu très pâle. Le noyau de type « en cocarde > est formé par une masse centrale arrondie ou ovalaire bien limitée, à chromatine homogène, siégeant au centre d'une bande de forme irrégulière beaucoup plus claire, blanche ou parfois rose pâle, généralement dépourvue de pigment. Le pigment est réparti dans l'ensemble du cytoplasme; il est noir, en forme de petites aiguilles de pin bien individualisées. Sa taille varie, mais le nombre de grains reste peu élevé (9-15). Le macrogamétocyte, très chromophile, a un cytoplasme bleu vif, parsemé de petites vacuoles contenant parfois de petites taches rouge brique. Le noyau, mal limité, a une chromatine granuleuse moins dense que celle du microgamétocyte.

\section{— Schizontes $(P l . I I, 1)$ :}

De très nombreux schizontes ont été trouvés sur les coupes (de 1 à 5 par coupe de $0,5 \mathrm{~mm}$ sur $0,25 \mathrm{~mm}$ ).

Le plus jeune élément observé mesure $12 \mu \mathrm{m} \times 9 \mu \mathrm{m}$. Il se développe dans une cellule hépatique, dont le noyau est repoussé en périphérie; il est légèrement ovoïde, entouré par une membrane fine et un espace de rétraction. Son cytoplasme bleu pâle est granuleux. Les noyaux sont de petite taille et arrondis; certains sont plus gros, aux contours irréguliers. La cellule parasitée persiste au cours de l'évolution du schizonte. Le noyau, de plus en plus repoussé à la périphérie, prend la forme d'un croissant ; le cytoplasme de la cellule-hôte forme une petite bande périphérique. A ce stade, le schizonte, de forme ovoïde, est très basophile. Ses noyaux sont gros, irréguliers et granuleux; la chromatine est très dense. Le cytoplasme parasitaire, bleu, est rempli de petites vacuoles.

Un schizonte presque mûr mesure $31 \mu \mathrm{m}$ sur $22 \mu \mathrm{m}$. Ses contours sont nets. Les noyaux présentent une structure très particulière. Ils sont formés d'une couronne de grains de chromatine avec un centre plus clair.

Nous n'avons observé aucune forme complètement mûre contenant des mérozoïtes.

\section{III. - Nycteria gabonensis n. sp.}

- Gamétocytes (Pl. I, 5, 6, 7):

Les infections étaient faibles et la plupart des parasites observés sont des gamétocytes mûrs. Seules trois formes jeunes ont été trouvées ; elles sont ovoïdes, avec un noyau de petite taille irrégulier et granuleux, une vacuole bordée par un cytoplasme en croissant. Cet aspect des parasites jeunes contraste avec le caractère compact de ceux des espèces décrites ci-dessus.

L'hématie parasitée par les gamétocytes est hypertrophiée ( $9 \mu \mathrm{m}$ en moyenne) et 
se colore en rose parfois foncé. Souvent déformée par le parasitisme, la cellule-hôte perd son aspect circulaire et prend une forme polyédrique irrégulière.

Les gamétocytes mûrs sont ronds et mesurent 7,65 $\mu \mathrm{m}$ de moyenne. Le microgamétocyte a un noyau volumineux ; sa chromatine est fine et homogène et ses limites bien définies; il est de type «diffus ». Le cytoplasme bleu-mauve entoure le noyau, sous forme d'un croissant ou d'une étroite couronne. Le pigment chevauche souvent le noyau ; de taille variable, il est formé de grains noirâtres. Le noyau du macrogamétocyte, beaucoup plus petit que celui du microgamétocyte est compact, ses contours sont irréguliers et sa chromatine est homogène. Un granule chromatinien (4) est présent de manière inconstante près du noyau. Le cytoplasme coloré en bleu vif contient de petites vacuoles. Le pigment présente le même aspect que celui du gamétocyte mâle, avec une concentration plus importante des grains autour du noyau.

\section{- Sehizonte :}

Un seul schizonte a été observé sur les coupes de foie. Immature, il est ovoïde, mesure $31 \mu \mathrm{m} \times 29 \mu \mathrm{m}$ et présente 2 lobes. Son cytoplasme est bleu, granuleux. Ses noyaux, mal colorés, sont irréguliers et de petite taille.

\section{IV. - Nycteria krampitzi n. sp.}

Rappel des principaux caractères morphologiques :

\section{- Gamétocytes mûrs :}

Ils mesurent 7,65 $\mu \mathrm{m}$ de moyenne et ne remplissent pas entièrement l'hématie parasitée, qui est généralement hypertrophiée; le noyau du microgamétocyte est de grande taille, de type diffus, de forme irrégulièrement carrée ou rectangulaire. Le pigment forme une couronne périphérique. Le macrogamétocyte a un noyau plus petit et est de forme irrégulière. Le pigment est limité à la zone annulaire du cytoplasme périphérique. Un granule chromatinien est présent chez le macrogamétocyte; il est inconstant chez le microgamétocyte.

- Schizontes (Pl. II, 5, 6, 7):

La plus jeune forme observée siège dans un hépatocyte dont le noyau est hypertrophié. Son cytoplasme est très basophile et les noyaux de grande taille sont granuleux. En vieillissant, le schizonte devient lobé, le cytoplasme toujours très basophile est parsemé de vacuoles rondes relativement grandes lui donnant un aspect spongieux. La membrane limitante s'épaissit.

Un schizonte proche de la maturité mesure $125 \mu \mathrm{m} \times 50 \mu \mathrm{m}$; il émet des

(4) Les gamétocytes de Rhinolophes capturés au mois d'août en étaient dépourvus, alors qu'il était fréquent en décembre. 
prolongements lobaires dans le tissu hépatique; le cytoplasme est plus clair que précédemment, les noyaux petits et arrondis. Une enveloppe épaisse, rose pâle, l'entoure et quelques rares macrophages se trouvent à proximité.

En 1973, Landau a décrit deux schizontes pulmonaires d'un type particulier chez un Rhinolophe ayant une parasitémie et chez lequel aucun schizonte hépatique n'avait été trouvé. Il s'agit de petits schizontes mesurant $25 \mu \mathrm{m}$, entourés par une capsule très épaisse $(8 \mu \mathrm{m})$ éosinophile. Le noyau de la cellule-hôte est accolé contre le rebord interne de la capsule. Une cavité centrale contient de 45 à 65 mérozoïtes allongés et de grande taille $(11 \mu \mathrm{m})$. Nous ne sommes pas certains que ces deux schizontes appartiennent au cycle du Nycteria, mais aucune autre espèce d'Hémosporidie n'étant présente chez cet hôte, nous le considérons provisoirement comme tel.

\section{V. - Nycteria medusiformis Garnham et Heisch, 1953}

- Gamétocytes (Pl. I, 8, 9,) :

Les formes très jeunes sont amiboïdes. Le noyau granuleux est constitué de plusieurs grains dispersés dans la masse chromatinienne aux contours irréguliers. Le cytoplasme bleu soutenu est parsemé de petites vacuoles rondes, qui lui donnent un aspect lacunaire. Des filaments rouges hérissent l'hématie hôte, qui ne subit pas d'autres modifications. Au cours de la maturation, le noyau s'étire en longueur, devient volumineux, et occupe le tiers du parasite, puis se rétracte et devient compact. Il perd sa position polaire pour devenir central. Le cytoplasme reste bleu mauve et vacuolisé presque jusqu'à la maturité complète. Le pigment, d'apparition tardive, est d'abord fin, jaune, très rassemblé près du noyau, et ne comporte que quelques grains bien séparés. Rapidement, il devient abondant, épais, dispersé et noirâtre.

\section{— Les microgamétocytes et macrogamétocytes mûrs :}

Ils sont ovalaires à frais et ronds sur frottis colorés séchés rapidement. Sensiblement de même taille, 7,75 $\mu \mathrm{m}$, ils n'envahissent pas la totalité de la cellule-hôte, qui reste très visible et fortement teintée en rose ; elle entoure le macrogamétocyte d'une couronne mince et persiste sous forme d'une zone irrégulière à un pôle du microgamétocyte. Les filaments de l'hématie sont moins nombreux que lorsque les parasites sont jeunes (1 à 4) et sont pour la plupart difficiles à déceler. Le cytoplasme bleu mauve du macrogamétocyte contraste avec celui du microgamétocyte (rose lilas). Les noyaux à chromatine homogène, ronds ou ovalaires, sont identiques dans les deux sexes. Dans certains gamétocytes femelles encore immatures, on distingue une masse chromatinienne accessoire mal limitée, qui a été interprétée par Garnham comme l'équivalent du centriole. Cette formation semble disparaître des gamétocytes mûrs. Le pigment est noir, constitué de gros grains bien individualisés et dispersés autour du noyau. 
- Schizontes (Pl. II, 8):

Nous avons retrouvé les principaux caractères morphologiques mis en évidence par Garnham et Heisch (1953) : structure complexe des noyaux et présence de lobes. Dans le parasite que nous avons étudié, cependant, les lobes au nombre de 2 à 3 ne font pas saillie vers l'extérieur, le schizonte restant généralement arrondi ou ovalaire.

Le plus jeune schizonte observé mesure $31 \times 21 \mu \mathrm{m}$, ses contours sont nets. Le noyau de la cellule-hôte est central, entouré par les lobes et hypertrophié
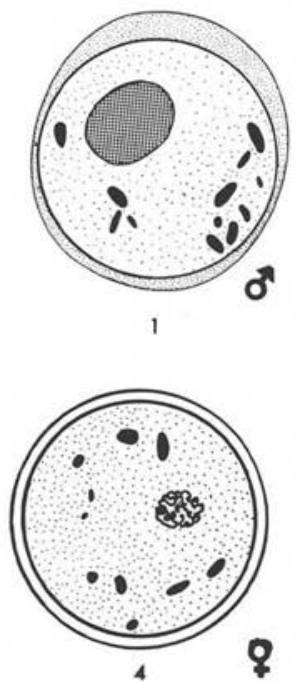

q

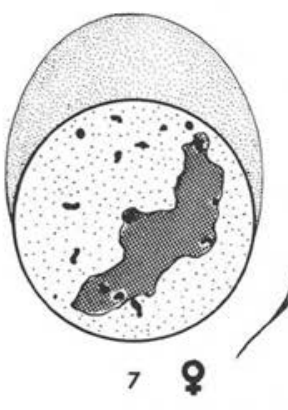

2

5
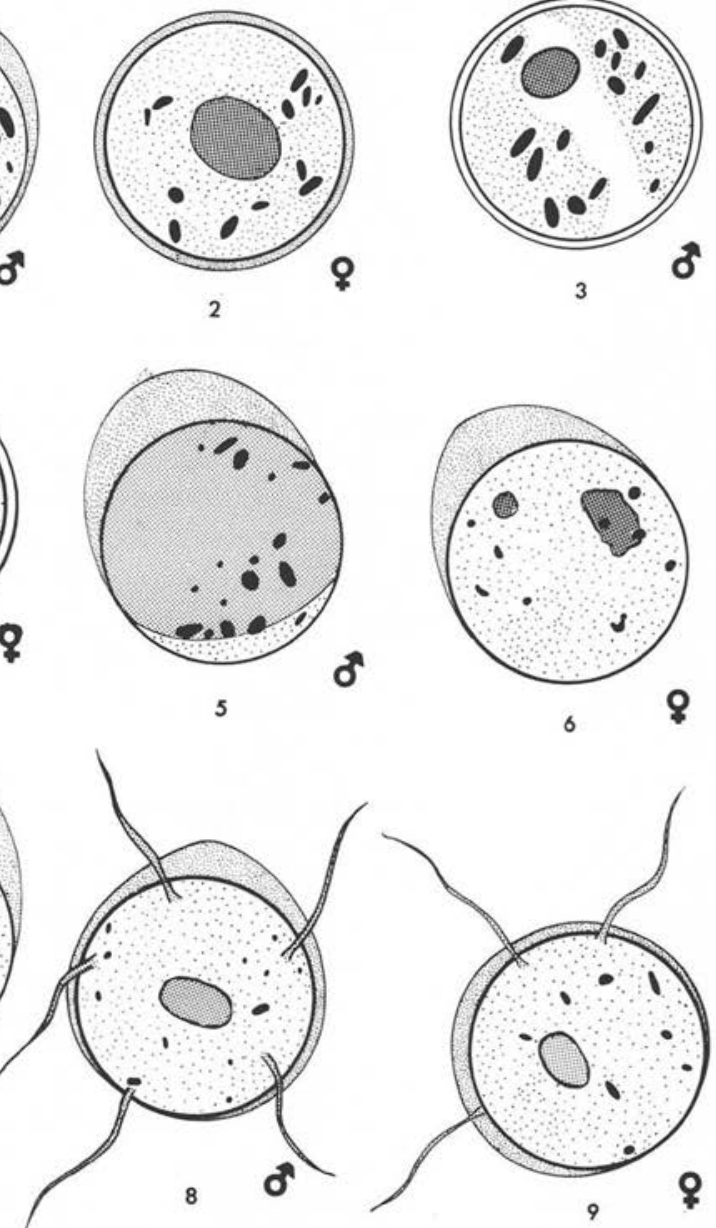
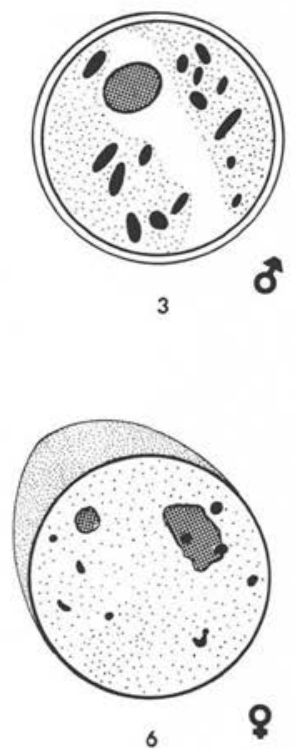

\% 
$(10,85 \times 7,75 \mu \mathrm{m})$; son nucléole est gros, la membrane nucléaire épaisse, et la chromatine rassemblée en petits amas périphériques le long de la membrane nucléaire. Le cytoplasme parasitaire, bleu intense, est granuleux. Les noyaux sont gros, irréguliers, et la chromatine dense. En mûrissant, les contours restent bien limités, les noyaux conservent le même volume, mais leur partie centrale devient plus claire et la chromatine se sépare en petites masses périphériques.

Un schizonte proche de la maturité mesure $70,4 \mu \mathrm{m} \times 46 \mu \mathrm{m}$ et présente un cytoplasme très clair, un nombre élevé de noyaux, qui restent assez gros, irréguliers et à chromatine dense.

B. - Discussion.

\section{I. - Le genre Nycteria : principaux caractères et diagnostic différentiel avec les autres genres}

- Le genre Nycteria est caractérisé par :

a) des gamétocytes de type «malariae »; les deux sexes sont de taille comparable, compacts, ne remplissant pas entièrement l'hématie-hôte; le pigment est grossier, formé de granules noirâtres bien individualisés, groupés en amas ou alignés. Deux types morphologiques existent: le premier a un microgamétocyte au noyau grand, diffus, occupant les trois-quarts du parasite, et un macrogamétocyte au noyau plus petit; le deuxième a, dans les deux sexes, un noyau petit, compact, bien limité, arrondi ou ovalaire, rarement entouré par une bande claire. Ces deux types bien identifiables et constants permettent de séparer les espèces de Nycteria en deux groupes (cf. plus loin).

Nous interprétons ces variations morphologiques des gamétocytes comme équivalentes à celles que l'on observe chez Hepatocystis, que nous avions subdivisé en sousgroupes «diffus» et «en cocarde». Bien que, chez Nycteria, les noyaux condensés n'aient pas un aspect «en cocarde » typique (anneau périphérique clair peu marqué), nous utilisons ici la même terminologie que pour Hepatocystis.

Le granule chromatinien accessoire est généralement associé au caractère « diffus : il est plus fréquent chez les macrogamétocytes que chez les gamétocytes mâles ;

b) des schizontes évoluant dans un hépatocyte et ayant généralement le type «aigu» (5). Les noyaux des formes jeunes sont de grande taille, avec une structure complexe, granulaire. Par la suite, ils deviennent plus petits, tout en conservant leur aspect granulaire. Proches de la maturité, ils sont arrondis et homogènes. Quelques

(5) Cytoplasme abondant et très basophile ; nombre de noyaux élevé par rapport au volume du schizonte; réaction cellulaire environnante absente ou faible. 

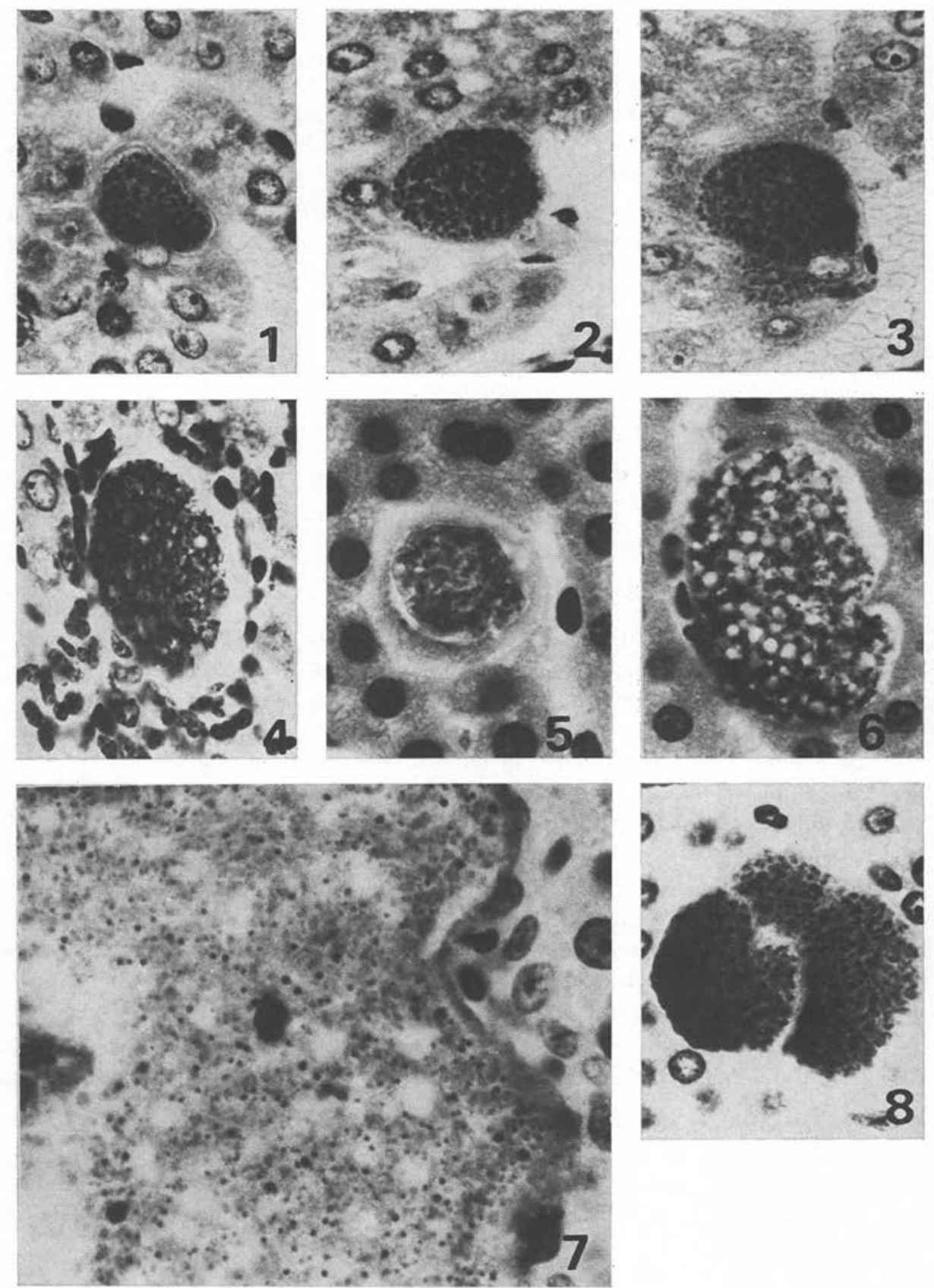

Planche II. Schizontes tissulaires: (1: N. erardi ; 2-3-4: N. houini; 5-6-7: N. krampitzi ; 
traînées d'aspect colloïdal et une enveloppe rose peuvent apparaître en fin d'évolution, mais elles n'atteignent jamais l'importance de celles des Hepatocystis;

c) des hôtes vertébrés (Rhinolophoïdea) appartenant aux genres Nycteris et Rhinolophus.

- Hepatocystis se différencie de Nycteria par :

a) les gamétocytes: ils sont de type «vivax» chez Hepatocystis, «malariae » chez Nycteria; ils remplissent l'hématie chez Hepatocystis et non chez Nycteria; le noyau du microgamétocyte est périphérique chez Hepatocystis et plutôt central chez Nycteria; le pigment est fin, abondant et diffus chez Hepatocystis, où il occupe toute la zone cytoplasmique, ses limites soulignant le contour du noyau qu'il ne chevauche jamais; il est plus grossier, les grains sont moins nombreux chez Nycteria, où sa distribution est irrégulière et recouvre souvent le noyau ;

b) la schizogonie : les schizontes hépatiques sont de grande taille chez Hepatocystis ( $>150 \mu \mathrm{m}$, et généralement beaucoup plus grands), de petite taille chez Nycteria $(<150 \mu \mathrm{m})$; la colloïde est généralement abondante chez les premiers, alors que ies seconds en sont presque dépourvus. Enfin, les noyaux d'Hepatocystis ont une chromatine homogène, alors que celle de Nycteria est granulaire.

- Polychromophilus diffère de Nycteria par:

a) les gamétocytes: microgamétocyte de teinte rose diffuse, avec un noyau mal limité ; pigment des gamétocytes plus fin et généralement plus dispersé ; forme ovalaire dans les frottis séchés lentement; granule chromatinien accessoire constant dans les deux sexes;

b) les schizontes: microschizontes réticulo-endothéliaux et macroschizontes pulmonaires et vasculaires hépatiques chez Polychromophilus, schizontes intra-hépatocytiques chez Nycteria.

\section{II. - Statut taxonomique des espèces}

Les six espèces de Nycteria connues jusqu'à présent peuvent être séparées en deux catégories selon la morphologie des gamétocytes: les parasites de Nycteris de type « en cocarde», et ceux de Rhinolophes de type « diffus».

a) Espèces de type «en cocarde »: N. medusiformis, N. houini, N. erardi.

$8:$ N. medusiformis). 1-2: jeunes schizontes intracellulaires avec grands noyaux ; $3:$ schizonte lobé ; 4 : cytomères, traces de colloïde et légère réaction histiomacrophagique ; 5 : jeune schizonte intracellulaire avec grands noyaux; 6: schizonte vacuolé caractéristique de $N$. ́rrampitzi ; 7 : détail d'un schizonte âgé avec petits noyaux et enveloppe épaisse ; 8 : schizonte polylobé à grands noyaux. 
- N. medusiformis se différencie aisément des deux autres espèces par la présence de filaments sur l'hématie parasitée, la plus grande taille de ses schizontes hépatiques et leur caractère fortement lobé.

- $N$. houini et $N$. erardi diffèrent par : la plus grande taille et le monomorphisme des noyaux des gamétocytes des deux sexes chez $N$. houini, leur dimorphisme et leur plus petite taille chez $N$. erardi; la plus grande taille des schizontes $(40 \mu \mathrm{m})$ et la présence d'un lobe chez $N$. houini, alors que les schizontes de $N$. erardi sont plus petits $(25 \mu \mathrm{m})$ et non lobés ;

b) Espèces de type « diffus » : ce sont Nycteria congolensis (Krampitz et Anciaux de Faveaux, 1960), N. gabonensis, N. krampitzi.

Elles sont indistingables dans le sang et diffèrent essentiellement par leurs schizontes exo-érythrocytaires.

$N$. krampitzi se distingue des deux autres espèces par le caractère très vacuolé des schizontes jeunes; $N$. congolensis diffère, en outre, du précédent par la capsule plus épaisse qui entoure les formes âgées. Nous n'avons observé qu'un seul schizonte relativement jeune de $N$. gabonensis. L'absence de vacuolisations permet de le différencier de $N$. krampitzi, mais aucun élément morphologique ne le sépare de $N$. congolensis. Nous lui avons cependant donné un statut séparé en raison des différences d'hôtes, de biotope, et de l'éloignement géographique, mais l'avenir montrera peut-être qu'en fait $N$. gabonensis et $N$. congolensis sont synonymes.

\section{III. - Place de Nycteria parmi les autres Hémosporidies}

La découverte de nombreuses espèces du genre Nycteria permet, non seulement de confirmer l'individualité du genre créé par Garnham et Heisch (1953), avec comme espèce type $N$. medusiformis, mais aussi de mieux préciser ses caractères et ses limites parmi les autres Haemoproteidae.

Les éléments les plus caractéristiques concernant la morphologie des gamétocytes et celle des schizontes hépatiques indiquent à quel point Nycteria est proche des Plasmodium du groupe malariae.

En reprenant nos hypothèses précédentes (Landau, Miltgen et Chabaud, 1976) sur le polyphylétisme en 3 lignées distinctes des Plasmodium humains, la lignée Nycteriamalariae paraît à tous points de vue plus primitive que la lignée Hepatocystis-vivax. La liste des hôtes comporte une majorité de Mammifères archaïques (Chiroptères, Lémuriens, Tragules, Primates), les plus archaïques d'entre eux étant africains ou malgaches (Chiroptères, Lémuriens).

(6) Les gamétocytes de Nycteria congolensis (Krampitz et Anciaux de Faveaux, 1960 ; Garnham, 1966) ont été découverts chez des Rhinolophus hildebrandti du Katanga et classés dans le genre Polychromophilus. Garnham (1966), après avoir trouvé, chez un $R$. $h$. eloquens du Kenya, un Haemoproteidae ayant des gamétocytes identiques à ceux du Katanga et des schizontes hépatiques de type Nycteria, a rassemblé les parasites de ces 2 localités sous le nom de Nycteria congolensis. Nous avons pu examiner des lames du matériel katangais que H. E. Krampitz a eu l'amabilité de nous communiquer: les gamétocytes ont, à notre avis, les caractères morphologiques d'une Nycteria. 
La morphologie des gamétocytes de type malariae est plus proche de celle des Hémosporidies de Reptiles et d'Oiseaux que celle des gamétocytes du type vivax; les schizontes hépatiques restent d'un type simple et ne présentent pas les hyperspécialisations remarquables du genre Hepatocystis.

La lignée Nycteria-malariae nous paraît donc un groupement naturel de type archaïque vraisemblablement originaire d'Afrique.

\section{Bibliographie}

Garnham P.C.C. (1966): Malaria parasites ant other Haemosporidia. T. 1. Blackwell Scientific Publications, Oxford, 114 p.

Garnham P.C.C., Heisch R. B. (1953): On a new blood parasite of insectivorous bats. Trans. R. Soc. trop. Med. Hyg., 47, 357-363.

Landau I. (1972): Diversité des mécanismes assurant la pérennité de l'infection chez les Sporozoaires coccidiomorphes. Mém. Mus. Nat. Hist. Nat., Nvlle sér. sér., A. Zool., 77, 1-62.

Landau I., Miltgen F., Chabaud A.-G. (1976): Les différents types de gamétocytes chez les Hémosporidies de Mammifères. Corrélations avec la morphologie des schizontes tissulaires. Hypothèses sur l'évolution du groupe. Ann. Parasitol. hum. comp., 51, 175-187.

Krampitz H. E., Anciaux de Faveaux F. M. (1960) : Über einiger Haemosporidien aus Fledermaüsen der Hohlen des Berglandes Katanga. Z. Tropenmed. Parasit., 11. 391-400. 